\title{
Prognostic factors in presurgical assessment of frontal lobe epilepsy
}

\author{
C H Ferrier, J Engelsman, G Alarcón, C D Binnie, C E Polkey
}

Institute of

Epileptology, King's College Hospital,

Denmark Hill, London

SE5 9RS, UK

C H Ferrier

J Engelsman

G Alarcón

C D Binnie

C E Polkey

Faculteit der

Geneeskunde,

Academic Medical

Center, University of

Amsterdam, The

Netherlands

C H Ferrier

$\mathrm{J}$ Engelsman

Departamento de Fisiología, Facultad de Medicina, Universidad Complutense, Madrid, Spain

G Alarcón

Correspondence to: Dr Gonzalo Alarcón, Department of Clinical Neurophysiology, King's College Hospital, Denmark Hill, London SE5 9RS, UK. Telephone 0044171346 5309; fax 0044171346 5308.

Received 26 March 1998 and in revised form

24 August 1998

Accepted 23 September 1998

\begin{abstract}
Objectives-To determine predictors for surgical outcome in the presurgical assessment of frontal lobe epilepsy.

Methods-Thirty seven patients were operated on for frontal lobe epilepsy between 1975 and 1996. Their medical records were reviewed for ictal semiology, age at onset, duration of the epilepsy, age at operation, preoperative interictal and ictal encephalographic findings, and abnormalities on neuroimaging and neuropsychological testing. In addition, type of resection and pathology were compared with surgical outcome.
\end{abstract}

Results-Univariate statistical analysis showed that the presence of a focal abnormality on neuroimaging was associated with favourable outcome. The presence of the following ictal findings was associated with poor outcome: autonomic manifestations, eye deviation, head version contralateral to the operated side, and bilateral or multifocal ictal onset. Fifteen patients had secondarily generalised interictal discharges and, interestingly, their presence was not associated with poor outcome. Multivariate logistic regression showed that the presence of a focal abnormality on neuroimaging was significantly associated with a favourable outcome while contralateral head version was the only variable significantly associated with poor surgical outcome.

Conclusions-A focal abnormality on neuroimaging was the only variable which was significantly associated with a favourable surgical outcome, whereas contralateral head version was the most significant predictor for a poor outcome. The presence of generalised discharges before surgery was not associated with poor outcome.

(F Neurol Neurosurg Psychiatry 1999;66:350-356)

Keywords: frontal lobe epilepsy; epilepsy surgery; presurgical assessment

Frontal lobe seizures are often resistant to medical treatment but may be amenable to surgery, accounting for as many as $18 \%$ of those patients who undergo surgery for epilepsy. ${ }^{12}$ However, outcome with regard to seizure control after frontal lobe surgery is generally poorer than after surgery for temporal lobe epilepsy. Although $56 \%$ of patients operated on for frontal lobe epilepsy experience a reduction in seizures, ${ }^{2}$ only a minority remain seizure free after a long follow up period. $^{3}$ The reasons behind these relatively poor results remain unclear. It has been suggested that the epileptogenic zone may be more extensive and difficult to identify in frontal lobe epilepsy, ${ }^{4}$ because of either widespread pathology or fast propagation. Although the symptomatology of frontal lobe seizures has been widely reported ${ }^{5-13}$ and the utilisation of multiple variables has been suggested to be useful in prediction of seizure relief after surgery for partial epilepsy, ${ }^{14-16}$ no clear features have been consistently reported as predictive of surgical outcome after frontal lobe surgery. Furthermore the lateralising and localising values of some simple clinical signs, such as head version, have remained controversial since the time of Hughlings Jackson. Most series correlating electroclinical or neuroimaging signs with outcome after frontal lobe surgery study a small number of patients either in isolation or within a larger series including patients with temporal lobe epilepsy or other types of extratemporal epilepsies. ${ }^{14}{ }^{16-24}$ Unfortunately the few large series of patients with frontal lobe epilepsy do not attempt to correlate ictal signs, pathology, type of resection, neuroimaging, or neuropsychology rigorously with surgical outcome. ${ }^{49} 25$

In this study we present findings from all patients who have had frontal lobectomies for epilepsy between 1975 and 1996 in the Maudsley Hospital and King's College Hospital, and correlate surgical outcome with age at onset, duration of epilepsy and age at operation, ictal symptomatology (clinical signs), interictal and ictal electroencephalographic findings, presence of abnormalities in neuroimaging, neuropsychology, type of resection, and pathology.

\section{Patients and methods}

PATIENTS

The clinical notes of all the patients who underwent a frontal lobectomy at our centre between 1975 and 1996 (42 patients) were reviewed retrospectively. Three patients had a follow up period shorter than 1 year and two had incomplete medical records. These five patients were excluded from the study, leaving 37 to be included.

The study comprised 18 males and 19 females. The age at onset of the epilepsy was between 0 and 19 years (mean 6.1 (SD 5.2)). The duration of the epilepsy at the time of operation ranged from 1 to 30 years (mean 10.6 (SD 7.9)). The age at operation was between 1 and 38 years (mean 16.8 (SD 10.4)). The period of follow up ranged from 1 to 19.5 years (mean 5.9 (SD 3.9)).

Although this was a retrospective study over two decades, the patients had all been treated 
by a team headed by the same neurosurgeon and two neurophysiologists. Detailed and standardised documentation was available.

OUTCOME

Surgical outcome was classified into four grades according to Engel et $a l^{26}$ : I (free of disabling seizures), II (almost seizure free, still having three or less seizures per year), III (worthwhile improvement remaining with more than three seizures per year), IV (no worthwhile improvement). Outcome grades I or II were considered favourable. Outcome grades III or IV were considered poor.

CLINICAL SIGNS

Full and detailed eye witnessed seizure descriptions were recorded by the neurosurgeon (CEP) in every patient. In addition, 15 patients have undergone video telemetry because there were discrepancies between different tests in the presurgical assessment and more detailed information about lateralisation or localisation was found to be necessary. Seizure description by the patient or relatives in all cases and telemetry videotapes or reports from 15 patients were used to evaluate the ictal symptoms. In the 15 patients who underwent video telemetry, ictal signs from video telemetry were used for analysis regardless of whether they were reported in the notes.

The presence of the following clinical features was recorded: aura, staring, automatisms (oroalimentary, bimanual/bipedal, cursive), motor signs (head version, eye deviation, focal twitches, posturing/dystonia), secondarily generalised seizures, vocalisation, autonomic manifestations (flushing, pallor, tachycardia, palpitations, changes in respiratory rhythm, mydriasis, salivation, frightened appearance), incontinence, postictal confusion, postictal dysphasia, and Todd's paralysis. Patients with head version to one side were further analysed as to whether the direction of the version was ipsilateral or contralateral to the operated side.

COMPARISON BETWEEN HISTORY AND TELEMETRY For the patients who had telemetry, the telemetry reports were compared with seizure descriptions in the clinical notes to check the reliability of ictal semiology reported in the history. This was done by counting the cases in which signs were present both in history and telemetry and whether their laterality was concordant.

\section{ELECTROENCEPHALOGRAPHIC FINDINGS}

Interictal

Of the patients studied, 33 had at least one preoperative scalp EEG record available for analysis. These were evaluated for the presence of (a) unilateral focal or unilateral multifocal epileptiform activity, $(b)$ bilateral synchronous discharges, (c) bilateral independent discharges, and (d) unilateral focal or multifocal epileptiform activity with bilateral synchronous discharges.

Ictal

From the patients who underwent intracranial or scalp telemetry, the ictal record was analysed for the extent of ictal onset and classified into focal, bilateral, or multifocal.

NEUROIMAGING

All patients had neuroimaging before the operation. Twenty patients had only CT and seven patients only MRI. Ten patients had CT and MRI. Neuroimaging findings were classified as normal, focal, multifocal, unilateral diffuse, or bilateral.

NEUROPSYCHOLOGY

Twenty five patients had neuropsychological assessment including full scale IQ, performance IQ, and verbal IQ. One of the following tests was performed: WAIS-R, WISC-R, WAIS, short-WAIS, WISC, or WPPSI-R. In right handed patients, a discrepancy between performance and verbal IQ greater than or equal to 15 points was considered as lateralising. If verbal IQ was larger than performance IQ a functional abnormality in the right hemisphere was inferred. If performance IQ was larger than verbal IQ, an abnormality in the left hemisphere was suspected.

PRESURGICAL ASSESSMENT AND RESECTION

The side of operation and extent and topography of the resection were decided on the basis of history, neuroimaging, neurophysiological findings (including intraoperative electrocorticography), and neuropsychology. In patients with normal neuroimaging, the side of resection was decided on the basis of history in addition to the presence of interictal EEG abnormalities or results from intracranial ictal telemetry. Four types of resection were performed:

(1) Total lobectomy: removal of the frontal lobe sparing the motor strip.

(2) Polar lobectomy: removal of anterior third or half of the frontal lobe.

(3) Parasagittal: resection of the mesial and superior aspects of the frontal lobe.

(4) Convexity: resection involving only the lateral aspect of the frontal lobe.

\section{PATHOLOGY}

After resection, material from all patients was sent for neuropathological examination. Pathological findings in resected specimens were divided into seven groups: cortical dysplasia, neoplasm, scarring, vascular malformation, no lesion, Rasmussen's encephalitis and other.

OUTCOME VERSUS TIME

Date of operation was correlated with surgical outcome, to establish changes in outcome with time. The surgical outcome was studied in the patients who were operated on between: (a) 1975 and 1985, (b) 1985 and 1991, and (c) 1991 and 1996.

\section{STATISTICAL METHODS}

The two tailed Mann-Whitney test was used to establish differences in continuous variables (age of onset, duration, age at operation) between the two outcome groups.

An initial univariate analysis using $\chi^{2}$ (one degree of freedom) or two tailed Fisher's exact 
Table 1 Sex, age at onset of epilepsy, duration of epilepsy, and age at operation in relation to surgical outcome

\begin{tabular}{llll}
\hline & \multicolumn{2}{l}{ Outcome } & \\
\cline { 2 - 4 } & Good $(n=20)$ & Poor $(n=17)$ & $\begin{array}{l}\text { Mann-Whitney } \\
\text { test }\end{array}$ \\
\hline Sex: & & & \\
$\quad$ Male & 10 & 8 & \\
$\quad$ Female & 10 & 9 & \\
Age at onset : & & & \\
$\quad$ Mean & 6.34 & 5.90 & $\mathrm{p}=0.73$ \\
$\quad$ SD & 5.04 & 5.42 & \\
$\begin{array}{l}\text { Duration: } \\
\quad \text { Mean }\end{array}$ & 9.71 & 11.69 & $\mathrm{p}=0.37$ \\
$\quad$ SD & 8.32 & 7.45 & \\
Age at operation: & & & \\
$\quad$ Mean & 16.05 & 17.59 & $\mathrm{p}=0.48$ \\
$\quad$ SD & 11.47 & 9.29 & \\
\hline
\end{tabular}

probability tests, was carried out to identify the clinical features most likely to be significantly associated with surgical outcome. Fisher's exact test was used when the expected number of patients in one cell of the contigency table was less than five. Similarly, the presence of a focal abnormality on neuroimaging was correlated with surgical outcome. Results were considered significant for $\mathrm{p}<0.05$.

At a second stage we designed a multivariate logistic regression model using surgical outcome as the dependent variable and the variables which seemed to correlate with surgical outcome after the univariate analysis as covariates. Covariates which showed prognostic value were identified with a forward stepwise approach. Independence of covariates was studied by incorporating their product (after coding as 0 or 1 ) into the multivariate logistic regression. The constant was forced to 0 in the regression equations as it provided a higher goodness of fit than non-zero constants (41.7 $v 37.0)$.

All statistical analysis was carried out with SPSS for Windows.

\section{Results}

PATIENTS

Out of the 37 patients studied, 12 had outcome grade I, eight grade II, nine grade III, and eight

Table 2 Relation between clinical signs and surgical outcome

\begin{tabular}{lrrr}
\hline & \multicolumn{2}{c}{ Outcome } & \\
\cline { 2 - 4 } & $\begin{array}{l}\text { Good } \\
\text { Symptom or sign }\end{array}$ & $\begin{array}{c}\text { Poor } \\
(n=17)\end{array}$ & p Value \\
\hline Aura & 9 & 11 & 0.231 \\
Staring & 12 & 10 & 0.942 \\
Vocalisation & 11 & 5 & 0.117 \\
Incontinence & 8 & 4 & 0.286 \\
Secondarily generalised seizures & 8 & 8 & 0.666 \\
Autonomic features & 6 & 11 & $0.035^{\star}$ \\
Oroalimentary automatisms & 5 & 2 & 0.416 \\
Cursive automatisms & 6 & 1 & 0.097 \\
Bimanual/bipedal automatisms & 8 & 9 & 0.431 \\
Contralateral head version & 1 & 9 & $0.002^{\star}$ \\
Ipsilateral head version & 4 & 2 & 0.667 \\
Eye deviation (any direction) & 5 & 10 & $0.037^{\star}$ \\
Myoclonias or clonic & & & \\
$\quad$ movements & 8 & 11 & 0.134 \\
Posturing & 13 & 11 & 0.985 \\
Postictal confusion & 6 & 7 & 0.478 \\
Postictal dysphasia & 3 & 3 & 1.000 \\
Todd's paralysis & 2 & 4 & 0.383 \\
\hline
\end{tabular}

The significance level accepted as suggesting statistical differences between favourable and poor outcome was $p<0.05$. Variables found to be associated with either favourable or poor outcome are indicated with an asterisk. grade IV. Thus, 20 patients (54\%) enjoyed a favourable outcome and $17(46 \%)$ had poor outcome. The age at onset of the epilepsy, the duration, and the age at operation for these two groups are listed in table 1 . None of these variables showed significant differences between the two outcome groups when analysed with a two tailed Mann-Whitney test.

\section{CLINICAL SIGNS}

The relation between several clinical variables and surgical outcome is shown in table 2. Contralateral head version, eye deviation, and autonomic manifestations during seizures were significantly associated with poor outcome.

Table 3 shows the relation between direction of head version and surgical outcome. There was a significant relation between contralateral version and poor outcome (Fisher's exact test, two tailed, $\mathrm{p}=0.002$ ).

COMPARISON BETWEEN HISTORY AND TELEMETRY Out of the 18 patients who had head version, 10 had video EEG telemetry of seizures. In two patients head version occurred to both sides in different seizures (one patient had head version to both sides in one seizure whereas the other patient had version of the head sometimes to the left and sometimes to the right in different seizures); they were excluded from the comparison between the direction of head version and outcome. In three further patients unilateral head version was not reported in the history but was seen in seizures recorded on telemetry. In the four patients in whom unilateral head version was previously reported in the medical notes and seen during the telemetry, the direction of head version was consistent. In one patient unilateral head version was reported in the history but not seen on telemetry.

Telemetry was carried out in 10 patients of the 24 who were considered to have ictal posturing and demonstrated posturing in nine. In five patients posturing was recorded in the notes and confirmed by telemetry. In four patients posturing was seen on telemetry, but not recorded in the medical history.

Nine patients who presented ictal myoclonias or clonic movements either on telemetry or in history had telemetry. Among these nine patients, such movements were seen on telemetry in seven patients and reported in the history of six patients. In four patients, jerking

Table 3 Direction of head version in relation to surgical outcome

\begin{tabular}{lll}
\hline & \multicolumn{2}{l}{ Outcome } \\
\cline { 2 - 3 } Head version & Good $(n=20)$ & Poor $(n=17)$ \\
\hline Contralateral & 1 & 9 \\
Ipsilateral & 4 & 2 \\
Inconsistent & 1 & 1 \\
No version & 14 & 5
\end{tabular}

The terms contralateral and ipsilateral refer to the direction of version with respect to the operated side. The term "inconsistent" refers to patients in whom head version occurred sometimes ipsilateral and sometimes contralateral to the operated side. When comparing contralateral head version with the rest, there is a very significant association between contralateral head version and poor outcome and between lack of contralateral head version and favourable outcome (contingency table $1,9,19,8$, Fisher's exact test, two tailed, $p=0.002$ ). 
was recorded in the notes and confirmed by telemetry. In two patients jerks were described in the notes but not seen on telemetry. In three patients the jerks were present on telemetry but not reported in the history. In all four patients in whom jerks were reported in the notes and described by telemetry, both methods agreed on the laterality of jerks.

It therefore seems that medical history reliably reported presence and laterality of convulsive ictal signs although with lower sensitivity than telemetry. In only one instance was a discrepancy in laterality found.

\section{ELECTROENCEPHALOGRAPHIC FINDINGS \\ Interictal}

Twenty one patients had unilateral focal or unilateral multifocal discharges in their preoperative interictal EEG, all of them lateralised to the operated side. One patient showed only a focal excess of slow activity with no epileptiform discharges in the right frontal area. This patient had a favourable surgical outcome. Interictal EEG findings were not predictive of surgical outcome (table 4).

Ictal

Among the 15 patients with ictal EEG on telemetry, nine had intracranial recordings with subdural or intracerebral electrodes. Eleven patients (including nine with intracranial EEG recordings) had focal seizure onset. Five of them had a favourable outcome whereas six patients had a poor outcome. Three patients had bilateral and one patient had multifocal seizure onset and these four patients had poor outcome. Two of these four patients had a focal abnormality on neuroimaging (oligodendroglioma, scar secondary to an abcess), one had multiple unilateral abnormalities and one had normal neuroimaging.

\section{NEUROIMAGING}

Twenty patients had a focal abnormality on neuroimaging. Seventeen patients had normal neuroimaging or presented non-focal abnormalities. Eleven of these had normal imaging of which four had a favourable outcome; two patients had multifocal abnormalities, both of them with a poor outcome; three patients had unilateral atrophy, one of them had a favourable outcome; one patient had bilateral atrophy and a favourable outcome.

Among the 20 patients who presented focal abnormalities on neuroimaging, 14 had favourable outcome. However only six of the 17 patients with no focal abnormalities on neuroimaging enjoyed a favourable outcome.

Table 4 Relation between surgical outcome and epileptiform activity on interictal scalp EEG

\begin{tabular}{lll}
\hline & \multicolumn{2}{l}{ Outcome } \\
\cline { 2 - 3 } Interictal EEG & Good $(n=16)$ & Poor $(n=17)$ \\
\hline Unilateral & 5 & 8 \\
Bilateral synchronous & 5 & 2 \\
Bilateral independent & 1 & 0 \\
$\begin{array}{l}\text { Unilateral + bilateral } \\
\text { synchronous }\end{array}$ & 4 & 4 \\
No epilepiform discharges & 1 & 3 \\
\hline
\end{tabular}

These proportions were significantly different $\left(\chi^{2}=4.46,1 \mathrm{df}, \mathrm{p}=0.035\right)$.

NEUROPSYCHOLOGY

Among the 25 patients who underwent neuropsychological testing, eight showed a lateralised deficit which suggested a dysfunction of the subsequently operated hemisphere in three patients. Among the three patients with psychometry lateralised to the operated side, two patients enjoyed favourable outcome and one patient had poor outcome. Among the five patients who showed neuropsychological preoperative deficits implicating the non-operated side, two had favourable outcome and three had poor outcome.

PRESURGICAL ASSESSMENT AND RESECTIONS Eleven patients had normal neuroimaging. Of these, four patients were operated on the basis of history and clear focal interictal EEG abnormalities, three of them were operated on before telemetric recordings were available and one did not have seizures during repeated 2 week telemetries. Seven patients were operated on the basis of findings from intracranial ictal recordings.

The relation between type of resection and surgical outcome was as follows: nine patients had a total lobectomy, four of whom enjoyed a favourable outcome; nine patients had a parasagittal resection, four with favourable outcome; eight patients had a resection confined to the convexity, four of whom had a favourable outcome; and 11 patients had a polar lobectomy, of whom eight had a favourable outcome. Although the number of patients for each type is small, patients who underwent a polar resection seem to have done better. After a polar lobectomy $73 \%$ had a favourable outcome whereas only $46 \%$ of patients had favourable outcome after a total frontal lobectomy, a parasagittal resection or a resection confined to the convexity of the frontal lobe.

In some patients functional procedures were performed in addition to resection at the time of the operation; one patient had in addition an anterior callosotomy and four had a procedure confined to the motor strip in addition to the resective surgery of the frontal lobe. The surgical outcome for the patient who had callosotomy was favourable. One of the four patients with multiple subpial transection had favourable outcome and three did poorly.

\section{PATHOLOGY}

The pathological findings in the resected material and the prognostic value of the different types of pathology in relation to surgical outcome are shown in table 5 . No particular pathology seems to be significantly associated with favourable or poor outcome. Nevertheless, vascular malformations seem to have better outcomes, whereas Rasmussen's encephalitis and "no lesion" have by comparison worse outcomes. Patients with "other pathology" included one with poor outcome and pathology report of "abnormal brain, with suspicion of some unusual neurodegenerative disorder" and two patients with favourable 
Table 5 Relation between pathology and surgical outcome

\begin{tabular}{lll}
\hline & \multicolumn{2}{l}{ Outcome } \\
\cline { 2 - 3 } Pathology & Good $(n=20)$ & Poor $(n=17)$ \\
\hline Cortical dysplasia & 10 & 7 \\
Neoplasm & 3 & 3 \\
Scarring & 2 & 2 \\
Vascular malformation & 3 & 0 \\
Rasmussen & 0 & 2 \\
Other pathology & 2 & 1 \\
No lesion found & 0 & 2 \\
\hline
\end{tabular}

outcome who were reported by the neuropathologist as Sturge-Weber syndrome, and extensive malformation reminiscent of tuberous sclerosis, partial pachygria (with microgyria), and dysplasia.

MULTIVARIATE LOGISTIC REGRESSION

The following covariates were used for the logistic regression model: autonomic features, contralateral head version, deviation of the eyes, and the presence of a focal abnormality on neuroimaging (Model $\chi^{2}=13.8,2 \mathrm{df}$, $\mathrm{p}=0.0010$, Goodness of fit $=41.7)$. The variables significantly associated with surgical outcome were presence of a focal abnormality on neuroimaging $(\mathrm{b}=1.32, \mathrm{SE}=0.57, \mathrm{p}=0.021, R$ $=0.25$ ) and contralateral head version $(b=-2.75, S E=1.12, p=0.014, R=-0.28)$. Both variables were independent $(p=0.19)$.

POSTOPERATIVE COMPLICATIONS

The most common postoperative complication was hemiparesis, which was seen in eight patients and gradually improved in all cases, probably because it was secondary to cerebral oedema. One patient developed monoplegia of the right arm due to extradural haematoma and gradually improved although a mild weakness persisted. Eight patients developed pyrexia which resolved with antibiotics in four. The other four patients required removal of the bone flap and some form of cranioplasty. Two patients had dysphasia which in one patient resolved whereas the other patient was left with a mild dysphasia secondary to a cerebrovascular accident. One patient had a CSF leak through the left nostril which required reopening of the craniotomy and patching of the dura with a graft of fascia lata. One patient developed buzzing sensations in the left ear. Eighteen patients did not have any postoperative complications.

OUTCOME VERSUS TIME

In the period from 1975 to 1985,12 patients (mean (SD) follow up period 9.5 (4.5) years) were operated on and six had favourable outcome. From 1985 to 1991, 13 patients (mean (SD) follow up period 5.0 (1.6) years) were operated on and only five had favourable outcome. Of the 12 patients (mean (SD) follow up period 3.4 (1.8) years who were operated on between 1991 and 1996 nine had favourable outcome. In the last group there were three patients with a follow up period of one to two years, all of them with a favourable surgical outcome.

\section{Discussion}

Some variables were significantly associated with surgical outcome. A focal abnormality on neuroimaging was associated with a good result. The presence of autonomic manifestations, eye deviation, contralateral head version, and bilateral or multifocal ictal onset was associated with poor outcome. A multivariate logistic regression model showed that only contralteral head version and the presence of a focal abnormality on neuroimaging had a significant prognostic value.

There was a significant association between version contralateral to the operated side and poor outcome. This may seem surprising as head version has traditionally been interpreted as suggestive of seizure onset contralateral to the direction of version since the early experiments involving electrical stimulation of the frontal lobe in primates and human patients. ${ }^{562728}$ There are several cortical and subcortical mechanisms by which both ipsilateral and contralateral head version can be induced (for an excellent discussion, see Ochs et $a l^{29}$ ). For instance, electrical stimulation of the frontal eye fields and supplementary motor cortex tends to induce contralateral version ${ }^{56}$ whereas stimulation of the superior temporal gyrus, premotor, and primary motor cortex is associated with version towards the stimulated side. ${ }^{27}{ }^{30}$ Thus, during a clinical frontal seizure, where widespread areas of the cortex may be rapidly activated, both ipsilateral and contralateral version could in principle be induced. In fact, some authors have mentioned the poor localising value of head version for establishing laterality in partial seizures, ${ }^{29} 3132$ whereas others found head version to be a significant lateralising sign. ${ }^{22} 23$ 33-37 Curiously, among the second group of authors, who found a significant association between head version and the location of seizure onset, there is a marked discrepancy as to whether head version occurs ipsilateral or contralateral to seizure onset. There are three main factors which seem to explain these differences: (a) the lobe involved at seizure onset, $(b)$ the quality of head version and its timing with respect to seizure onset or secondary generalisation, and (c) the methodology used to identify seizure onset. Ochs et al studied the direction of head version with simultaneous intracranial or scalp electroencephalographic recordings in 106 seizures from 43 patients with partial epilepsy. ${ }^{29}$ Ipsilateral head version was found in 53 seizures and contralateral head version was seen in 41 seizures, this difference was not significant. However, in the 17 seizures with clear frontal onset in the EEG, the head deviated to the ipsilateral side in 14 seizures and to the contralateral side in only three seizures $\left(\chi^{2}\right.$ $=7.12,1 \mathrm{df}, \mathrm{p}<0.01)$, suggesting that at least in frontal lobe epilepsy, head version may have localising value to the ipsilateral side. Similarly, Quesney found that in 56 seizures with ictal electroencephalographic changes strictly confined to one frontal lobe, ipsilateral head version was seen in 36 seizures and contralateral version in $20 .^{38}$ However, McLachlan found that out of 25 patients regarded as having 
frontal lobe epilepsy by electrocencephalographic and neuroimaging criteria, 24 had contralateral head version and only one had ipsilateral head version according to reliable witnesses or to the patients themselves. ${ }^{33} \mathrm{It}$ could be argued that findings from history taking could not be sufficiently reliable, as it is based on witnesses' accounts. However, when favourable surgical outcome rather than diagnostic procedures is used to lateralise seizure onset, Quesney et al found in a retrospective study of "pure" frontal lobe epilepsy (composed of patients rendered seizure free after frontal lobe surgery), that unilateral head version was a lateralising sign to the side contralateral to version if present early in the seizure and without any other motor manifestation. ${ }^{36}$ It has also been suggested that in patients with frontal lobe seizures who remain conscious during head version, often occurring as the initial ictal manifestation, the direction is usually contralateral to the epileptic focus. ${ }^{38}$

Discrepancies regarding version could be explained by the findings of Kernan et al and Fakhoury and Abou-Khalil who found that early isolated version in partial seizures which finishes before secondary generalisation tends to be ipsilateral whereas version taking place more than 120 seconds into the seizure or just before secondary generalisation is more likely to be contralateral. ${ }^{3435}$ This can be explained if we assume that widespread areas of the ipsilateral frontal lobe are recruited during the development of secondary generalisation, including those areas responsible for contralateral head version. Such fast propagation responsible for contralateral version could be associated with poorer outcome, as in the case in temporal lobe epilepsy. ${ }^{39}$

The vast majority of patients had unilateral focal or unilateral multifocal discharges in their interictal EEG. This is not surprising considering that this was one of the criteria used in deciding to perform surgery. ${ }^{1}{ }^{40}$ However, 15 patients had secondarily generalised or bilaterally synchronous discharges. Interestingly, such discharges were not associated with poor outcome, by contrast with the findings by Lieb et al in temporal lobe epilepsy. ${ }^{41}$ These authors reported that out of nine patients operated on for temporal lobe epilepsy who presented with bilaterally synchronous discharges in their scalp interictal EEG, none had a favourable outcome, perhaps suggesting a frontal onset or early frontal involvement. In fact, it has long been established that secondarily generalised discharges can arise from localised areas of the frontal lobes ${ }^{42}$ and also that they could spread very rapidly and almost explosively. ${ }^{43}$ Indeed, frontal lobe epilepsies with generalised bilateral synchrony can be improved by frontal resections $^{44}$ and as many as $65 \%$ of patients who remained seizure free 5 years after surgery for frontal lobe epilepsy had bilaterally synchronous discharges in their preoperative interictal EEG. ${ }^{45}$

The four patients with bilateral or multifocal ictal onset had poor outcome, regardless of the presence of a focal abnormality on neuroimag- ing in two subjects. Similar findings have recently been reported elsewhere ${ }^{24}$ and emphasise that the presence of localised abnormalities on neuroimaging, although being a predictor for good outcome, does not necessarily imply a favourable result, particularly when discrepancies between structural and functional tests exist.

In our centre outcome has improved since 1991. Although follow up periods are necessarily shorter for patients operated on more recently, this is probably not the main factor contributing to the improvement of outcome as only three patients among those operated on later than 1991 had a follow up period shorter than 2 years. The most important factors in improving surgical outcome are likely to be advances in surgical techniques and presurgical assessment, including the advent of neuroimaging.

The present study has implications for presurgical assessment of frontal lobe epilepsy. It seems that in addition to localising signs and symptoms, prediction of outcome requires the identification of factors which specifically appear to be associated with favourable outcome. For instance, the presence of autonomic manifestations during seizures was associated with poor outcome. Given the rapid seizure propagation in frontal lobe epilepsy, early symptoms can arise from areas located far from or contralateral to the initial functional abnormality, and the association between such findings and outcome may not be obvious. Thus some predictors of outcome should be found empirically as, in addition to the location and extent of the epileptogenic zone, they may also depend on the pathways and speed of propagation. Because in most centres, the number of patients with frontal lobe epilepsy is relatively small, statistically significant associations are difficult to obtain although strong, nearly significant trends are often seen. Convergence of multiple methodologies in localising functional and structural abnormalities could be the best predictor for outcome and it appears that convergence of both structural and functional techniques is desirable to obtain favourable outcome. Prospective studies considering several diagnostic methods in all patients are necessary.

This study was supported by the Dutch Nationaal Epilepsie Fonds (NEF, De Macht van het Kleine). The authors are grateful to Dr J L Fernandez Torre (London), Professor D A Bosch (Amsterdam), and Dr J Overweg (Heemstede) for their support and advice.

1 Binnie CD, Polkey CE. Surgery for epilepsy. In: Kennard C, ed. Recent advances in clinical neurology. London: Churchill Livingstone, 1992;7:55-93.

2 Rasmussen T. Tailoring of cortical excisions for frontal lobe epilepsy. Can f Neurol Sci 1991;18:606-10.

3 Hajek M. Extratemporal epilepsies: surgical results. fournal of Epilepsy 1988;1:103-19.

4 Talairach J, Bancaud J, Bonis A, et al. Surgical therapy for frontal lobe epilepsies. In: Chauvel P, Delgado-Escueta AV, et al, ed. Advances in neurology. New York: Raven Press, 1992;57:707-32

5 Penfield W. Functional localization in the cerebral cortex. In: Penfield W, Jasper H. Epilepsy and the functional anatomy of the human brain, 1st edition. London: Churchill, 1954:41155.

6 Penfield W. Somatic motor seizures. In: Penfield W, Jasper H. Epilepsy and the functional anatomy of the human brain. $1 \mathrm{st}$ H. Epilepsy and the functional anatomy of

7 Chauvel P, Bancaud J. The spectrum of frontal lobe seizures: with a note of frontal lobe syndromatology. In: 
Wolf P, ed. Epileptic seizures and syndromes. London: John Libbey, 1994:331-4.

8 So NK. Supplementary motor area epilepsy: the clinical syndrome. In: Wolf P, ed. Epileptic seizures and syndromes. London: John Libbey, 1994:299-317.

9 Bancaud J, Talairach J. Clinical semiology of frontal lobe seizures. In: Chauvel P, Delgado-Escueta AV, Halgren E, et al, eds. Advances in neurology (vol 57). Frontal lobe seizures and epilepsies. New York: Raven Press, 1992;57:3-59.

10 Williamson PD, Spencer SS. Clinical and EEG features of complex partial seizures of extratemporal origin. Epilepsia 1986;27(suppl 2):S46-63.

11 Morris III HH, Frontal lobe epilepsies. In: Lüders H, ed. Epilepsy surgery. New York: Raven Press, 1991:157-65.

12 Trottier S, Chauvel P, Bancaud J. Seizures from the precentral and the premotor areas. I. Symptomatology as a function of onset topography. Epilepsia 1988;29:206-7.

13 Saint-Hilaire JM, Neilleux F, Giard M, et al. Frontal lobe epileptic manifestations studied with depth electrodes. Epilepsia $1988 ; 29: 207-8$.

14 Dodrill CB, Wilkus RJ, Ojemann GA, et al. Multidisciplinary prediction of seizure relief from cortical resection surnary prediction of seizure relief
gery. Ann Neurol 1986;20:2-12.

15 Armon C, Radtke RA, Friedman AH, et al. Predictors of outcome of epilepsy surgery: multivariate analysis with validation. Epilepsia 1996;37:814-21.

16 Guldvog B, Loyning Y, Hauglie-Hanssen E, et al. Predictive factors for success in surgical treatment for partial epilepsy: a multivariate analysis. Epilepsia 1994;35:566-78.

17 Boon PA, Williamson PD, Fried I, et al. Intracranial, intraaxial, space-occupying lesions in patients with intractable partial seizures: an anatomoclinical, neuropsychologi$\mathrm{cal}$, and surgical correlation. Epilepsia 1991;32:467-76.

18 Adler J, Erba G, Winston KR, et al. Results of surgery for extratemporal partial epilepsy that began in childhood. Neurology 1991;48:133-40.

19 Davies KG, Weeks RD. Cortical resections for intractable epilepsy of extratemporal origin: experience with seventeen epilepsy of extratemporal origin: experience with sevent
cases over 11 years. Br $\mathcal{F}$ Neurosurg 1993;7:343-53.

20 Laskowitz DT, Sperling MR, French JA, et al. The syndrome of frontal lobe epilepsy: characteristics and syndrome of frontal lobe epilepsy: character

21 Cukiert A, Olivier A, Andermann F. Post-traumatic frontal lobe epilepsy with structural changes: excellent results after cortical resection. Can f Neurol Sci 1996;23:114-7.

22 Bleasel A, Kotagal P, Kankirawatana P, et al. Lateralizing value and semiology of ictal limb posturing and version in temporal lobe and extratemporal epilepsy. Epilepsia 1997; 38:168-74

23 Chee MW, Kotagal P, Van Ness PC, et al. Lateralizing signs in intractable partial epilepsy: blinded multiple-observer analysis. Neurology 1993;43:2519-25.

24 Swartz BE, Delgado-Escueta AV, Walsh GO, et al. Surgical oucomes in pure frontal lobe epilepsy and foci that mimic them. Epilepsy Res 1998;29:97-108.

25 Cascino GD, Jack Jr CR, Parisi JE, et al. MRI in the presurgical evaluation of patients with frontal lobe epilepsy and children with temporal lobe epilepsy: pathologic correlation and prognostic importance. Epilepsy Res 1992;11:51-9.
26 Engel J Jr, Van Ness PC, Rasmussen TB, et al. Outcome with respect to epileptic seizures. In: Engel J Jr, ed. Surgical treatment of the epilepsies. New York: Raven Press, 1993: 609-21.

27 Ferrier D. The localization of function in the brain. London: Proceedings of the Royal Society of London, 1874

28 Penfield W, Erickson T. Epilepsy and cerebral localization. Springfield, IL: Charles C Thomas, 1941:51-2.

29 Ochs R, Gloor P, Quesney F, et al. Does head-turning during a seizure have lateralizing or localizing significance? Neurology 1984;34:884-90.

30 Rasmussen T, Penfield W. Movement of head and eyes from stimulation of human frontal cortex. Research Publications of the Association for Research in Nervous and Mental Disease 1948;27:346-61.

31 Robillard A, Saint-Hilaire JM, Mercier M, et al. The lateralizing and localizing value of adversion in epileptic seizures. Neurology 1983;33:1241-2.

32 Quesney LF. Clinical and EEG features of complex partial seizures of temporal lobe origin. Epilepsia 1986; 27(suppl.2):S27-45.

33 McLachlan RS. The significance of head and eye turning in seizures. Neurology 1987;37:1617-19.

34 Fakhoury T, Abou-Khalil B. Association of ipsilateral head turning and dystonia in temporal lobe seizures. Epilepsia 1995;36:1065-70.

35 Kernan JC, Devinsky O, Luciano DJ, et al. Lateralizing significance of head and eye deviation in secondary generatized tonic-clonic seizures. Neurology 1993;43:1308-10.

36 Quesney LF, Constain M, Fish DR, et al. The clinical differentiation of seizures arising in the parasagittal and anterolaterodorsal frontal convexities. Arch Neurol 1990;47:677-9.

37 Wyllie E, Lüders $\mathrm{H}$, Morris $\mathrm{HH}$, et al. The lateralizing significance of versive head and eye movements during epileptic seizures. Neurology 1986; 36:606-11.

38 Quesney LF. Seizures of frontal lobe origin. In: Pedley TA, Meldrum BS, ed. Recent advances in epilepsy. London: Meldrum BS, ed. Recent advances

39 Lieb JP, Engel J Jr, Babb TL. Interhemispheric propagation time of human hippocampal seizures in relationship to surtime of human hippocampal seizures in relati
gical outcome. Epilepsia 1986;27:286-93.

40 Polkey CE, Binnie CD. Assessment and selection of candidates for surgical treatment. Epilepsia 1995;36(suppl 1):S41-5.

41 Lieb JP, Engel J Jr, Gevins A, et al. Surface and deep EEG correlates of surgical outcome in temporal lobe epilepsy. Epilepsia 1981;22:515-38.

42 Loiseau P. Childhood absence epilepsy. In Roger J, Bureau M, Dravet $\mathrm{C}$, et al, ed. Epileptic syndromes in infancy, childhood and adolescence. London: John Libbey 1992:135-50.

43 Williamson PD. Frontal lobe seizures: problems of diagnosis and classification. In: Chauvel P, Delgado-Escueta AV, et al, eds. Advances in neurology. New York: Raven Press, 1992;57:289-309.

44 Stefan H, Rasmussen T, Quesney LF. Uni- or bifrontal epileptogenic areas: clinical relevance of secondary bilateral synchrony. Epilepsia 1988;29:211.

45 Rasmussen T. Characteristics of a pure culture of frontal lobe epilepsy. Epilepsia 1983;24:482-93. 